\title{
Therapeutic Activity of Lenalidomide in Mantle Cell Lymphoma and Indolent Non-Hodgkin's Lymphomas
}

\author{
Marco Gunnellini and Lorenzo Falchi \\ S. C. Oncohematology, Perugia University, S. Maria, 05100 Terni, Italy \\ Correspondence should be addressed to Lorenzo Falchi, lorenzo.falchi@gmail.com \\ Received 16 February 2012; Revised 29 March 2012; Accepted 16 April 2012 \\ Academic Editor: Umberto Vitolo
}

Copyright ( $) 2012$ M. Gunnellini and L. Falchi. This is an open access article distributed under the Creative Commons Attribution License, which permits unrestricted use, distribution, and reproduction in any medium, provided the original work is properly cited.

\begin{abstract}
Mantle cell lymphoma (MCL) comprises 3-10\% of NHL, with survival times ranging from 3 and 5 years. Indolent lymphomas represent approximately $30 \%$ of all NHLs with patient survival largely dependent on validated prognostic scores. High response rates are typically achieved in these patients with current first-line chemoimmunotherapy. However, most patients will eventually relapse and become chemorefractory with poor outcome. Alternative chemoimmunotherapy regimens are often used as salvage strategy and stem cell transplant remains an option for selected patients. However, novel approaches are urgently needed for patients no longer responding to conventional chemotherapy. Lenalidomide is an immunomodulatory drug with activity in multiple myeloma, myelodisplastic syndrome and chronic lymphoproliferative disorders. In phase II studies of indolent NHL and MCL lenalidomide has shown activity with encouraging response rates, both as a single agent and in combination with other drugs. Some of these responses may be durable. Optimal dose of lenalidomide has not been defined yet. The role of lenalidomide in the therapeutic armamentarium of patients with indolent NHL or MCL will be discussed in the present paper.
\end{abstract}

\section{Introduction}

Non-Hodgkin's lymphomas (NHLs) are a heterogeneous group of lymphoid malignancies. The annual incidence of NHL in the United States is estimated to be $4.5 \%$ of all cancers, and they account for $3 \%$ of annual cancer-related deaths [1]. From a clinical and therapeutic standpoint, these neoplasias are subdivided into aggressive and indolent forms. Indolent lymphomas represent approximately $30 \%$ of all NHLs. Prognosis is correlated with the stage of the disease at the time of diagnosis, as well as to the international prognostic index (IPI) or other IPI-derived scores [2-5]. The current therapeutic approach for indolent NHL is based on the use of chemoimmunotherapy. Intensive treatments such as high-dose chemotherapy with autologous stem cell transplantation (ASCT) are typically reserved for relapsing patients whose disease is still chemosensitive [1].

Mantle cell lymphoma (MCL) comprises approximately 3 to $10 \%$ of NHL. It is a heterogeneous clinical entity with four recognized morphologic variants (i.e., classical, blastoid, pleomorphic and small cell, marginal zone-like). The small cell variant tends to be an indolent lymphoma, whereas both the blastoid and pleomorphic variants are associated with a clinical aggressive course.

However, the majority (80\%) of MCLs show intermediate characteristics. Thus, the median survival of the majority of patients is in the range of 3 to 5 years, and very few patients are cured [2].

MCL patients typically respond well to initial treatment with an overall response rate of approximately $90 \%$. The addition of rituximab to conventional chemotherapy has even improved both quality and durability of responses either in newly diagnosed or relapsed disease $[6,7]$.

However, most patients will eventually relapse, with shorter and shorter disease-free intervals, and will require multiple different therapeutic interventions during the course of their disease $[8,9]$. For this reason, there is a need for new effective agents with novel mechanisms of action to be tested in these patients. 


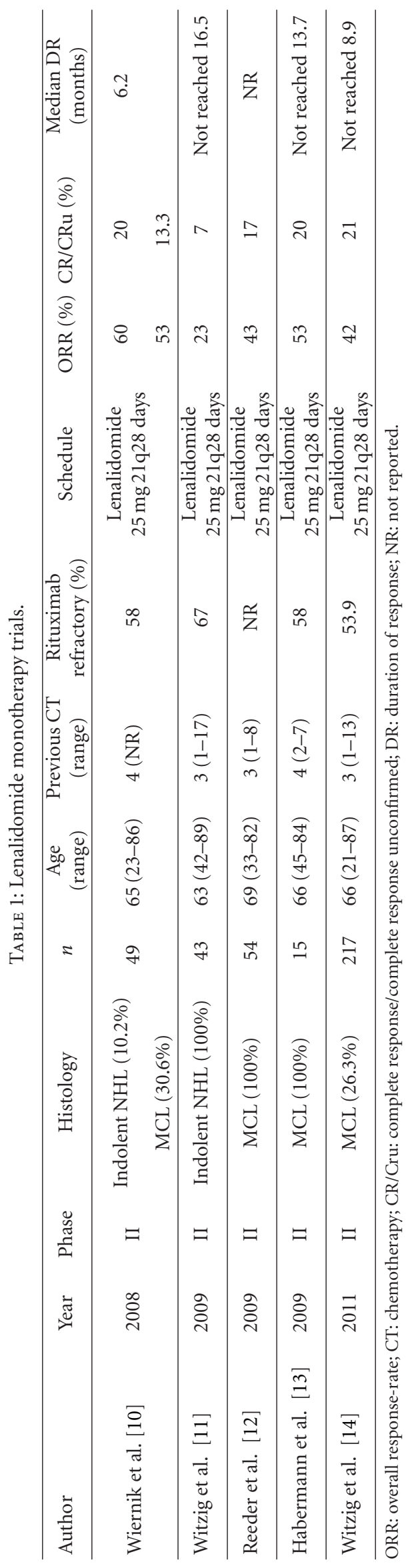


TABLE 2: Ongoing lenalidomide (L) monotherapy trials.

\begin{tabular}{lccccc}
\hline Name & Phase & Age & Histology & Drugs & Status \\
\hline NCT00875667 & II & $>18$ ys & Relapsed or refractory MCL & L & Ongoing and recruiting \\
NCT00737529 & II & $>18$ ys & Relapsed or refractory MCL & L & Ongoing and recruiting \\
NCT00179673 & II & $>18$ ys & Relapsed or refractory indolent NHL & L & Terminated \\
\hline
\end{tabular}

L: lenalidomide.

\section{Rationale for and Development of Lenalidomide in Lymphoproliferative Disorders}

Lenalidomide is an immunomodulatory drug (IMiD), derived from thalidomide, with increased potency and fewer side effects compared to its parent molecule. This agent has shown impressive clinical activity in patients with multiple myeloma (MM) [15] and has proven effective in chronic lymphocytic leukemia (CLL) [16] and T-cell lymphoma [17]. Preclinical models and preliminary clinical data also indicate significant antitumor activity of lenalidomide in B-cell malignancies $[18,19]$.

The mechanism of action of lenalidomide includes both immunomodulatory and nonimmunomodulatory effects [20-24]. It inhibits the production of proinflammatory cytokines (TNF- $\alpha$, IL-1, IL-6, and IL-12) and enhances that of anti-inflammatory cytokine (IL-10) resulting in an increase of the tumor-cell apoptosis [20-22]. Lenalidomide also induces tyrosine phosphorylation of CD28, providing a costimulatory signal to T-cell activation by antigen-presenting cells via the B7 pathway [20-22]. IMiDs can decrease the expression of the angiogenic factors VEGF and IL-6 leading to a reduction of growth and survival of tumor cells [20-25]. Lenalidomide increases the number and $\mathrm{Fc}-\gamma$ receptor-mediated cytotoxicity of NK cells with an as-yet unclear mechanism of action [20]. Importantly, lenalidomide has also shown direct antiproliferative activity, in the absence of immune effectors, by decreasing erk $1 / 2$ and Akt2 and by inducing G0-G1 cell cycle arrest through inhibition of CDK2 activity [20-23]. Finally, in MM, lenalidomide has been shown in vitro to alter the microenvironment by downregulating cell surface adhesion molecules such as ICAM-1, VCAM-1, and E -selectin and inhibiting the adhesion of MM cell lines to the bone marrow stromal cells [20,21].

\section{Lenalidomide Monotherapy in Relapsed/Refractory Indolent and Mantle Cell Lymphoma}

Oral lenalidomide monotherapy produces durable responses in patients with NHL with a manageable toxicity profile (Table 1). In a pilot study of relapsed/refractory aggressive NHL, also including 15 and 5 stage III follicular lymphoma (FL) patients, lenalidomide induced an objective response rate of $35 \%$ with $12 \%$ complete responses/unconfirmed complete responses [10]. Patients enrolled in the study had received a median of 4 prior therapies. Fifty eight percent of patients were rituximab refractory. The most frequent G3 toxicity was neutropenia. A dose reduction was necessary in $18(37 \%$ ) patients ( 9 patients to $20 \mathrm{mg}, 5$ patients to $15 \mathrm{mg}$, 3 patients to $10 \mathrm{mg}$, and 1 patient to a $5 \mathrm{mg}$ daily dose). Eight patients $(16 \%)$ discontinued treatment because of adverse events. In a second trial [11] of heavily pretreated indolent NHL patients (median number of prior lines $3(1-17)$ ), single-agent lenalidomide resulted in an ORR of $23 \%(27 \%$ in follicular and $22 \%$ in small lymphocytic lymphoma). Median duration of response was not reached with a followup of 15 to 28 months. Median PFS for the whole group was 4.4 months (95\% CI, 2.5-10.4). The most frequent G3 toxicities were hematological (neutropenia, thrombocytopenia, and anemia). Seventeen patients (40\%) had a dose reduction ( 6 patients to $20 \mathrm{mg}, 3$ patients to $15 \mathrm{mg}, 6$ patients to $10 \mathrm{mg}$, and 2 patients to a $5 \mathrm{mg}$ daily dose). Eight patients (19\%) discontinued treatment because of adverse events, and 1 died on treatment due to sepsis. In another study of relapsed/refractory MCL, lenalidomide showed an overall response rate (ORR) of $43 \%$. Twenty six percent of the patients had received stem cell transplantation and 32\% had been exposed to bortezomib. ORR in these two groups was $53 \%$ and $57 \%$, respectively. The most common grade 3 or 4 adverse event was neutropenia (43\%) [12]. In a smaller study including 15 patients, $58 \%$ of whom being rituximab refractory, objective responses were achieved in $53 \%$ of cases with a $20 \%$ complete remission (CR) rate $[13,26]$. Eight patients $(53 \%)$ had a dose reduction, but only 1 patient discontinued treatment. Finally, Witzig et al. [14] treated 217 aggressive relapsed/refractory NHLs, including $26 \%$ of patients with MCL. Median number of prior chemotherapy lines was 3 (1-13). MCL patients showed an ORR of $42 \%$ with a median progression-free survival (PFS) not reached. Fifty three $(53 \%)$ of patients required dose reduction (37 patients to $20 \mathrm{mg}, 11$ patients to $15 \mathrm{mg}, 9$ patients to $10 \mathrm{mg}$, and 10 patients to a $5 \mathrm{mg}$ daily dose), and $23 \%$ discontinued lenalidomide. Most G3-G4 toxicities recorded were hematological (41\% neutropenia, 19\% thrombocytopenia, and $9.2 \%$ anemia). Ongoing trials of single-agent lenalidomide in refractory indolent NHL and MCL are summarized in Table 2. While in untreated patients a lenalidomide daily dose of $25 \mathrm{mg}$ may be appropriate (see Table 1), in patients with relapsed or refractory disease, particularly if they are elderly and/or suffering from other comorbidities, a lower dose (15 to $20 \mathrm{mg}$ ) appears to be a wiser choice. Of note, because of the proliferation of phase II studies with different starting doses, and the dose-escalating design of several ongoing lenalidomide trials, a dose that is considered "reasonable" may not necessarily be the optimal one. 
TABLE 3: Lenalidomide containing regimens in relapsed/refractory indolent and mantle cell lymphoma.

\begin{tabular}{lccccccccc}
\hline Author & Year & Phase & Histology & $n$ & Age (range) & Previous CT & Combination & ORR (\%) & CR (\%) \\
\hline Wang et al. [27] & 2007 & I/II & MCL & 15 & $73(62-84)$ & $2(1-7)$ & RL & $83^{*}$ & $17^{*}$ \\
Dutia et al. [28] & 2009 & II & Indolent NHL & 15 & $60(50-91)$ & $4(1-11)$ & RL & 83.3 & 41 \\
Zaja et al. [29] & 2011 & II & MCL & 33 & $68(51-80)$ & $3(2-7)$ & LD & 52 & 24 \\
\hline
\end{tabular}

${ }^{*}$ : data from patients enrolled in lenalidomide $20 \mathrm{mg} /$ daily arm. No response was obtained for lower dosage.

CT: chemotherapy; ORR: overall response rate; CR: complete response; RL: rituximab and lenalidomide; LD: lenalidomide and dexamethasone.

TABLE 4: Ongoing lenalidomide-based regime trials.

\begin{tabular}{lccccc}
\hline Name & Phase & Age & Histology & Drugs & Status \\
\hline NCT01419795 & II & $>18$ ys & Relapsed or refractory NHL after allo-SCT & RL & Ongoing and recruiting \\
NCT00238238 & II & $>18$ ys & Relapsed follicular NHL & RL & Ongoing and recruiting \\
NCT00633594 & I/II & $>18$ ys & Relapsed or refractory MCL & RVL & Ongoing and recruiting \\
NCT00553644 (27) & II & $>18$ ys & Relapsed or refractory MCL & LV & Ongoing and recruiting \\
\hline
\end{tabular}

RL: rituximab, lenalidomide; RVL: rituximab, bortezomib, lenalidomide; LV: lenalidomide, bortezomib.

\section{Lenalidomide in Combination for Relapsed/Refractory Indolent and MCL}

The Fc portion of rituximab mediates ADCC. Lenalidomide increases $\mathrm{Fc}-\gamma$ receptors on $\mathrm{NK}$ cell surface enhancing rituximab-mediated ADCC. Many trials have therefore evaluated the two drugs in combination (Tables 3 and 4). In a phase I/II study [27] of rituximab $\left(375 \mathrm{mg} / \mathrm{m}^{2}\right.$ weekly for 4 doses) and escalating doses of lenalidomide (from 10 to $25 \mathrm{mg}$ daily on days 1-21 of 28-day cycles for a total of 6 cycles) in relapsed or refractory MCL, no responses were observed in the $10 \mathrm{mg}$ and $15 \mathrm{mg}$ groups, while patients receiving $20 \mathrm{mg}$ daily achieved an ORR of $83 \%$, including $17 \%$ of complete responses. At the dose of $25 \mathrm{mg}$, a G3 hypercalcemia and a lethal neutropenic fever were observed. The recommended lenalidomide daily dose to be used in combination with rituximab in phase II trials was therefore established to be $20 \mathrm{mg}$. Dutia et al. [28] tested rituximab ( $375 \mathrm{mg} / \mathrm{m}^{2}$ weekly for 4 doses plus 4 doses if no CR was reached) and lenalidomide (20 mg daily on days $1-21$ of 28day cycles) in heavily pretreated patients with indolent NHL. Treatment proved active and well tolerated (no dose reductions nor discontinuations were reported), particularly in patients with rituximab-refractory FL (response rate of $55 \%)$. In a recent phase II study that enrolled patients with MCL and either relapsed/refractory disease or ineligibility to intensive treatment, lenalidomide $25 \mathrm{mg}$ daily for days 1-21 plus dexamethasone (40 mg on days 1, 8, 15, and 22) were given as postinduction consolidation therapy for 3 (patients in CR) or up to 12 (patients in partial remission/stable disease-PR/SD) cycles. Treatment was discontinued at CR or unacceptable toxicity. The study enrolled 33 patients. Median number of prior treatments was $3(2-7)$. After a median followup of 16 months, median PFS and OS were 12 and 20 months, respectively, with median response duration of 18 months. Treatment was well tolerated, the most frequent toxicity being neutropenia (grade 3 in 25\%, grade 4 in 28\% of patients), leading to treatment interruption in two patients. Overall 9 serious adverse events were recorded, including one therapy-related fatal acute respiratory insufficiency [29].
Phase II trials are underway to test different combinations, notably including bortezomib plus lenalidomide [30].

\section{Lenalidomide in Combination for Untreated Indolent and Mantle Cell Lymphoma}

The combination of rituximab and lenalidomide has also been tested in previously untreated patients with indolent NHL and MCL (Tables 5 and 6). In an ongoing study of 30 patients with advanced-stage indolent NHL and indication for treatment, [31] rituximab $\left(375 \mathrm{mg} / \mathrm{m}^{2}\right.$ on day 1 of each 28 -day cycle) and lenalidomide (20 mg/day on days $1-21)$ for 6 cycles produced an ORR of $86 \%$ and an overall response rate (CRR) of $79 \%$. Only 2 patients required treatment discontinuation due to toxicities leading the investigators to expand the originally planned accrual and include a total of 110 patients. In a study of 75 patients with indolent NHL [32], the same combination induced responses in $90 \%$ of patients with a $66 \%$ CRR. Only 5 patients discontinued treatment within the first two cycles due to toxicity. In a recent phase I trial [33], escalating doses of lenalidomide (from 5 to $25 \mathrm{mg}$ once daily on days 1-14) were associated to R-CHOP21 (rituximab $375 \mathrm{mg} / \mathrm{m}^{2}$, cyclophosphamide $750 \mathrm{mg} / \mathrm{m}^{2}$, doxorubicin $50 \mathrm{mg} / \mathrm{m}^{2}$, vincristine $1.4 \mathrm{mg} / \mathrm{m}^{2}$ on day 1 , and prednisone $100 \mathrm{mg} / \mathrm{m}^{2}$ days $1-5$ every 21 days) for 6 cycles in untreated B-cell lymphomas. Lenalidomide $25 \mathrm{mg}$ was established as the recommended dose. The most frequent toxicity was hematological (grade 3-4 neutropenia in 59\% of patients), 6 patients experienced cycle delay, and 5 discontinued treatment, but no toxic death occurred.

Strategies to build on the use of lenalidomide as a single agent appear the avenue to pursue. The role of dexamethasone is marginal, if any. On the contrary, the association of lenalidomide and rituximab appears to be feasible and shows encouraging activity in untreated and previously treated patients with indolent and MCL. The combination of lenalidomide with chemoimmunotherapy regimens such as R-CHOP is attracting, but both its feasibility and efficacy need to be tested in further prospective trials. Finally, 
TABLE 5: Lenalidomide containing regimens in untreated indolent and mantle cell lymphoma.

\begin{tabular}{lcccccccc}
\hline Author & Year & Phase & Histology & $n$ & Age (range) & Combination & ORR (\%) & CR (\%) \\
\hline Fowler et al. [31] & 2010 & II & Indolent NHL & 30 & $56(36-77)$ & RL & 86 & 79 \\
Samaniego et al. [32] & 2011 & II & Indolent NHL & 75 & $57(35-84)$ & RL & 90 \\
Tilly et al. [33] & 2011 & I & NHL & 27 & NR & RL-CHOP & 96 & 74 \\
\hline
\end{tabular}

ORR: overall response rate; CR: complete response; NR: not reported; RL: rituximab and lenalidomide; RL-CHOP: rituximab, lenalidomide, cyclophosphamide, doxorubicin, vincristine, prednisone.

TABLE 6: Ongoing lenalidomide-based regime trials.

\begin{tabular}{lccccc}
\hline Name & Phase & Age & Histology & Drugs & Status \\
\hline NCT01415752 & II & $>60$ ys & Untreated MCL & RBV + RL & Ongoing and recruiting \\
NCT01316523 & II & $>18$ ys & Untreated indolent NHL & RL & Ongoing and recruiting \\
NCT00695786 & II & $>18$ ys & Untreated indolent NHL & RL & Ongoing and recruiting \\
FIL R2-B & II & $>18$ ys & Untreated indolent NHL & RBL & Ongoing and recruiting \\
\hline
\end{tabular}

RBV: rituximab, bendamustine, bortezomib; RL: rituximab, lenalidomide; RBL: rituximab, bendamustine, lenalidomide.

TABLE 7: Ongoing lenalidomide-maintenance trials.

\begin{tabular}{lccccc}
\hline Name & Phase & Age & Histology & Drugs & Status \\
\hline NCT01035463 & I/II & $>19$ ys & Relapsed or refractory NHL & R-BEAM + ASCT + mL & Ongoing and recruiting \\
NCT01035463 & I/II & $>18$ ys & Relapsed or refractory NHL & R-BEAM + ASCT + mL & Ongoing and recruiting \\
NCT01254578 & I & $>18$ ys & High-risk hematologic cancers after Allo-SCT & mL & Ongoing and recruiting \\
NCT01045928 & I/II & $>18$ ys & NHL & R + mL & Ongoing not recruiting \\
NCT01021423 & III & $>18$ ys & Untreated MCL & FCR or R-CHOP + mL & Ongoing not recruiting \\
IIL MCL0208 & III & $18-60$ ys & Untreated MCL & R-BEAM + ASCT + mL & Ongoing and recruiting \\
\hline
\end{tabular}

R-BEAM: rituximab, BCNU, etoposide, ara-C, melphalan; ASCT: autologous stem cell transplantation; mL: lenalidomide maintenance; R: rituximab; FCR: fludarabine, cyclophosphamide; rituximab; R-CHOP: rituximab, cyclophosphamide, doxorubicin, vincristine, prednisone.

the exploration of lenalidomide in other chemotherapy-free combination regimens is particularly fascinating and eagerly awaited.

\section{Lenalidomide as Maintenance Therapy for MCL}

With the aim of increasing disease control and survival, some authors have proposed a postinduction maintenance strategy for patients with MCL. One agent that proved successful in this context is rituximab [34]. Twenty-two untreated MCLs not candidate for autologous stem cell transplantation were treated with a maximum of 6 cycles repeated every 28 days of modified R-hyper-CVAD (rituximab, hyperfractionated cyclophosphamide, vincristine, doxorubicin, and dexamethasone) followed by rituximab maintenance (weekly doses every 6 months for a total of 4 courses). ORR and CRR were impressive ( $77 \%$ and $64 \%$, resp.). In a recently presented multicenter phase III trial [35], 560 untreated elderly (>60 ys) patients not eligible for high-dose therapy were randomized to receive R-CHOP or rituximab, fludarabine, and cyclophosphamide followed by a maintenance phase with either rituximab or interferon-alfa. Rituximab maintenance doubled the remission duration compared to IFN (57\% versus $26 \%$ at 4 years, resp., $P=0.0109$ ). Not surprisingly, hematologic grade 3-4 toxicity was higher in the IFN arm. Overall survival did not differ between both maintenance arms $(P=0.17)$. Another randomized phase III trial evaluated the efficacy of lenalidomide versus placebo as maintenance therapy after first-line induction in patients with MCL not candidates for intensive treatment. Lenalidomide was given orally at the dose of $15 \mathrm{mg}$ daily on days $1-21$ every 28 days for 2 years, up to either disease progression or unacceptable toxicity, whichever occurred first. Only 9 patients (4 in CR and 5 in PR) were randomized (4 in the lenalidomide maintenance arm and 5 in the placebo). Two patients discontinued treatment due to toxicity and disease progression in the treatment and placebo arm, respectively. The study was prematurely terminated, and most analyses were not performed. A phase I/II Scandinavian trial [36] is ongoing in which lenalidomide is combined with rituximab $\left(375 \mathrm{mg} / \mathrm{m}^{2}\right.$ on day 1$)$ and bendamustine $\left(90 \mathrm{mg} / \mathrm{m}^{2}\right.$ on days $1-2)$ as induction in untreated elderly ( $>65$ years) MCL patients. Six 28 -day induction cycles are followed by seven 28-day cycles of maintenance lenalidomide $(25 \mathrm{mg}$ daily on days 1-21). Recently, Ahmadi et al. [37] investigated the safety and efficacy of lenalidomide and rituximab in relapsed/refractory indolent or mantle cell lymphoma. Forty five sequential patients received two 28-day treatment cycles of lenalidomide $10 \mathrm{mg}$ every day and four weekly doses of rituximab $375 \mathrm{mg} / \mathrm{m} 2$ in cycle 3 with (cohort 1) or without 
(cohort 2) weekly dexamethasone. In stable and responding patients, lenalidomide and dexamethasone (cohort 1) or lenalidomide alone (cohort 2) was continued until disease progression or unacceptable toxicity (median number of prior therapies was $3(1-7))$. Thirty five patients were evaluable for response. At a median followup of 11.8 months, PFS was 73\% (95\% CI: 53-86\%), and ORR was 60\% (12 CR; $9 \mathrm{PR})$. ORR did not differ between cohort 1 and cohort $2, P=$ 0.5 . Half of the patients temporarily suspended treatment, while 2 discontinued therapy. Several other trials of lenalidomide maintenance in patients with untreated or relapsed/ refractory NHL are underway (Table 7).

\section{Conclusions}

Lenalidomide is an immunomodulatory agent with remarkable activity in a variety of lymphoproliferative disorders. Its value is well established in multiple myeloma, and increasing evidence supports its role in the management of CLL patients. Phase II data are also delineating a role for lenalidomide in NHL, including MCL and indolent NHLs. Response rates are encouraging with response lasting 6.2 to 16.5 months in relapsed NHL when used alone. The drug is generally tolerated with hematological adverse events being the most common toxicity, and no unexpected toxicities in numerically limited trials the optimal dose of lenalidomide in maintenance or in combination with other agents remains to be defined. Single-agent lenalidomide $25 \mathrm{mg}$ may be appropriate in young untreated patients, while a lower dose (15 to $20 \mathrm{mg}$ ) should be considered in relapsed/refractory elderly patients. The route is traced out, but informative, randomized phase III trials with careful study design and adequate patient numbers will be necessary to define the role of lenalidomide in the therapeutic armamentarium of patients with NHL.

\section{References}

[1] K. Kaunshansky, M. Lichtman, E. Beutler, T. Kipps, J. Prchal, and U. Seligsohn, Williams Hematology, McGrow-Hill, 8th edition, 2011.

[2] S. H. Swerdlow, E. Campo, N. L. Harris et al., WHO Classification of Tumours of Haematopoietic and Lymphoid Tissues, WHO Press, 4th edition, 2008.

[3] S. A. van de Schans, E. W. Steyerberg, M. R. Nijziel, G. J. Creemers, M. L. Janssen-Heijnen, and D. J. van Spronsen, "Validation, revision and extension of the follicular lymphoma international prognostic index (FLIPI) in a population-based setting," Annals of Oncology, vol. 20, no. 10, pp. 1697-1702, 2009.

[4] C. H. Geisler, A. Kolstad, A. Laurell et al., "The mantle cell lymphoma international prognostic index (MIPI) is superior to the international prognostic index (IPI) in predicting survival following intensive first-line immunochemotherapy and autologous stem cell transplantation (ASCT)," Blood, vol. 115, no. 8, pp. 1530-1533, 2010.

[5] L. H. Sehn, B. Berry, M. Chhanabhai et al., "The revised international prognostic index (R-IPI) is a better predictor of outcome than the standard IPI for patients with diffuse large B-cell lymphoma treated with R-CHOP," Blood, vol. 109, no. 5, pp. 1857-1861, 2007.
[6] H. Schulz, J. E. Bohlius, S. Trelle et al., "Immunochemotherapy with rituximab and overall survival in patients with indolent or mantle cell lymphoma: a systematic review and metaanalysis," Journal of the National Cancer Institute, vol. 99, no. 9, pp. 706-714, 2007.

[7] S. Sachanas, G. A. Pangalis, T. P. Vassilakopoulos et al., "Combination of rituximab with chlorambucil as first line treatment in patients with mantle cell lymphoma: a highly effective regimen," Leukemia \& Lymphoma, vol. 52, no. 3, pp. 387-393, 2011.

[8] M. J. Kersten, "Radioimmunotherapy in follicular lymphoma: some like it hot...," Transfusion and Apheresis Science, vol. 44, no. 2, pp. 173-178, 2011.

[9] A. K. Gopal, J. G. Rajendran, T. A. Gooley et al., "Highdose $\left[{ }^{131} \mathrm{I}\right]$ tositumomab (anti-CD20) radioimmunotherapy and autologous hematopoietic stem-cell transplantation for adults $\geq 60$ years old with relapsed or refractory B-cell lymphoma," Journal of Clinical Oncology, vol. 25, no. 11, pp. 13961402, 2007.

[10] P. H. Wiernik, I. S. Lossos, J. M. Tuscano et al., "Lenalidomide monotherapy in relapsed or refractory aggressive nonHodgkin's lymphoma," Journal of Clinical Oncology, vol. 26, no. 30, pp. 4952-4957, 2008.

[11] T. E. Witzig, P. H. Wiernik, T. Moore et al., "Lenalidomide oral monotherapy produces durable responses in relapsed or refractory indolent non-Hodgkin's lymphoma," Journal of Clinical Oncology, vol. 27, no. 32, pp. 5404-5409, 2009.

[12] C. B. Reeder, T. E. Witzig, P. L. Zinzani et al., "Efficacy and safety of lenalidomide oral monotherapy in patients with relapsed or refractory mantle-cell lymphoma: results from an international study (NHL-003)," Journal of Clinical Oncology, vol. 27, no. 15, supplement, abstract e19504, 2009.

[13] T. M. Habermann, I. S. Lossos, G. Justice et al., "Lenalidomide oral monotherapy produces a high response rate in patients with relapsed or refractory mantle cell lymphoma," British Journal of Haematology, vol. 145, no. 3, pp. 344-349, 2009.

[14] T. E. Witzig, J. M. Vose, P. L. Zinzani et al., "An international phase II trial of single-agent lenalidomide for relapsed or refractory aggressive B-cell non-Hodgkin's lymphoma," Annals of Oncology, vol. 22, no. 7, pp. 1622-1627, 2011.

[15] M. Roussel, T. Facon, P. Moreau, J. L. Harousseau, and M. Attal, "Firstline treatment and maintenance in newly diagnosed multiple myeloma patients," Recent Results in Cancer Research, vol. 183, pp. 189-206, 2011.

[16] M. Gentile, A. G. Recchia, E. Vigna et al., "Lenalidomide in the treatment of chronic lymphocytic leukemia," Expert Opinion on Investigational Drugs, vol. 20, no. 2, pp. 273-286, 2011.

[17] G. Dueck, N. Chua, A. Prasad et al., "Interim report of a phase 2 clinical trial of lenalidomide for T-cell non-hodgkin lymphoma," Cancer, vol. 116, no. 19, pp. 4541-4548, 2010.

[18] Z. Qian, L. Zhang, Z. Cai et al., "Lenalidomide synergizes with dexamethasone to induce growth arrest and apoptosis of mantle cell lymphoma cells in vitro and in vivo," Leukemia Research, vol. 35, no. 3, pp. 380-386, 2011.

[19] A. A. Chanan-Khan and B. D. Cheson, "Lenalidomide for the treatment of B-cell malignancies," Journal of Clinical Oncology, vol. 26, no. 9, pp. 1544-1552, 2008.

[20] V. Kotla, S. Goel, S. Nischal et al., "Mechanism of action of lenalidomide in hematological malignancies," Journal of Hematology and Oncology, vol. 2, article 36, 2009.

[21] N. Reddy, F. J. Hernandez-Ilizaliturri, G. Deeb et al., "Immunomodulatory drugs stimulate natural killer-cell function, alter cytokine production by dendritic cells, and inhibit angiogenesis enhancing the anti-tumour activity of rituximab in 
vivo," British Journal of Haematology, vol. 140, no. 1, pp. 3645, 2008.

[22] A. A. Chanan-Khan and B. D. Cheson, "Lenalidomide for the treatment of B-cell malignancies," Journal of Clinical Oncology, vol. 26, no. 9, pp. 1544-1552, 2008.

[23] D. H. Chang, N. Liu, V. Klimek et al., "Enhancement of liganddependent activation of human natural killer $\mathrm{T}$ cells by lenalidomide: therapeutic implications," Blood, vol. 108, no. 2, pp. 618-621, 2006.

[24] L. Wu, M. Adams, T. Carter et al., "Lenalidomide enhances natural killer cell and monocyte-mediated antibody-dependent cellular cytotoxicity of rituximab-treated $\mathrm{CD} 20^{+}$tumor cells," Clinical Cancer Research, vol. 14, no. 14, pp. 4650-4657, 2008.

[25] L. Zhang, Z. Qian, Z. Cai et al., "Synergistic antitumor effects of lenalidomide and rituximab on mantle cell lymphoma in vitro and in vivo," American Journal of Hematology, vol. 84, no. 9, pp. 553-559, 2009.

[26] P. L. Zinzani, T. E. Witzig, J. M. Vose et al., "Confirmation of the efficacy and safety of lenalidomide oral monotherapy in patients with relapsed or refractory mantle-cell lymphoma: results of an international study (NHL-003)," Blood, vol. 112, abstract 262, 2008.

[27] M. Wang, L. Fayad, F. Hagemeister et al., "A phase I/II study of lenalidomide (Len) in combination with rituximab $(\mathrm{R})$ in relapsed/refractory mantle cell lymphoma (MCL) with early evidence of efficacy," Journal of Clinical Oncology, vol. 25, no. 18, supplement, abstract 8030, 2007.

[28] M. Dutia, I. DeRoock, K. Chee et al., "R2: preliminary results of a phase II study of lenalidomide and rituximab in relapsed/ refractory indolent non-Hodgkin's lymphoma (NHL)," Blood, vol. 114, abstract a1679, 2009.

[29] F. Zaja, S. De Luca, U. Vitolo et al., "Salvage treatment with lenalidomide and dexamethasone in relapsed/refractory mantle cell lymphoma: clinical results and effects on microenvironment and neoangiogenic biomarkers," Haematologica, vol. 97, no. 3, pp. 416-422, 2012.

[30] V. A. Morrison, S. Jung, J. L. Johnson et al., "A phase II trial of bortezomib plus lenalidomide for relapsed/refractory mantle cell lymphoma (MCL) (CALGB 50501)," Journal of Clinical Oncology, vol. 29, abstract TPS223, 2011.

[31] N. H. Fowler, P. McLaughlin, F. B. Hagemeister et al., "Complete response rates with lenalidomide plus rituximab for untreated indolent B-cell non-Hodgkin's lymphoma," Journal of Clinical Oncology, vol. 28, no. 15, supplement, abstract a8036, 2010.

[32] F. Samaniego, F. Hagemeister, P. Mclaughlin et al., "High response rates with lenalidomide plus rituximab for untreated indolent B-cell non-Hodgkin lymphoma, including those meeting GELF criteria," Journal of Clinical Oncology, vol. 29, abstract a8030, 2011.

[33] H. Tilly, F. Morschhauser, G. A. Salles et al., "Phase I study of escalating doses of lenalidomide combined with R-CHOP (R2-CHOP) for front-line treatment of B-cell lymphomas," Blood, vol. 118, no. 21, abstract a1632, 2011.

[34] V. P. Kenkre, W. L. Long, J. C. Eickhoff et al., "Maintenance rituximab following induction chemo-immunotherapy for mantle cell lymphoma: long-term follow-up of a pilot study from the wisconsin oncology network," Leukemia \& Lymphoma, vol. 52, no. 9, pp. 1675-1680, 2011.

[35] J. C. Kluin-Nelemans, E. Hoster, J. Walewski et al., "R-CHOP versus R-FC followed by maintenance with rituximab versus interferon-alfa: outcome of the first randomized trial for elderly patients with mantle cell lymphoma," Blood, vol. 118, abstract a439, 2011.

[36] M. Jerkeman, A. Kolstad, A. Laurell et al., "Lenalidomide, bendamustine, and rituximab as first-line therapy for patients $>65$ years with mantle cell lymphoma: the nordic lymphoma group MCL4 (LENA-BERIT) trial," Journal of Clinical Oncology, vol. 28, no. 18, supplement, abstract a18567, 2010.

[37] T. Ahmadi, E. A. Chong, A. Gordon et al., "Phase II trial of lenalidomide-rituximab $+/$ - dexamethasone in relapsed or refractory indolent B-cell or mantle cell lymphomas resistant to rituximab," Blood, vol. 118, abstract a266, 2011. 


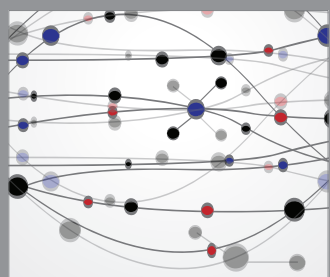

The Scientific World Journal
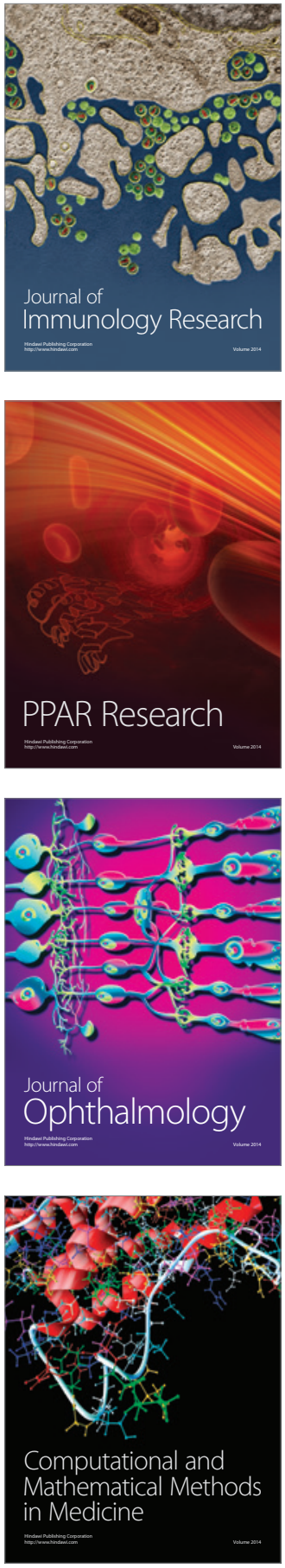

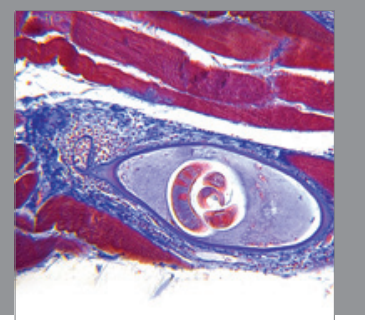

Gastroenterology

Research and Practice
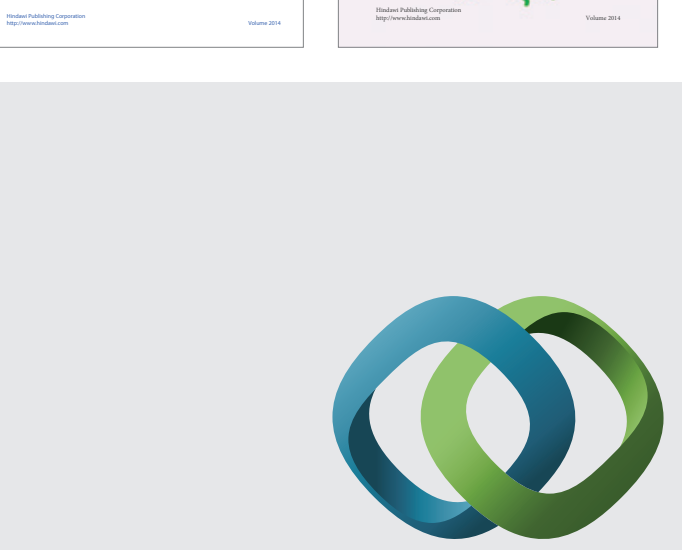

\section{Hindawi}

Submit your manuscripts at

http://www.hindawi.com
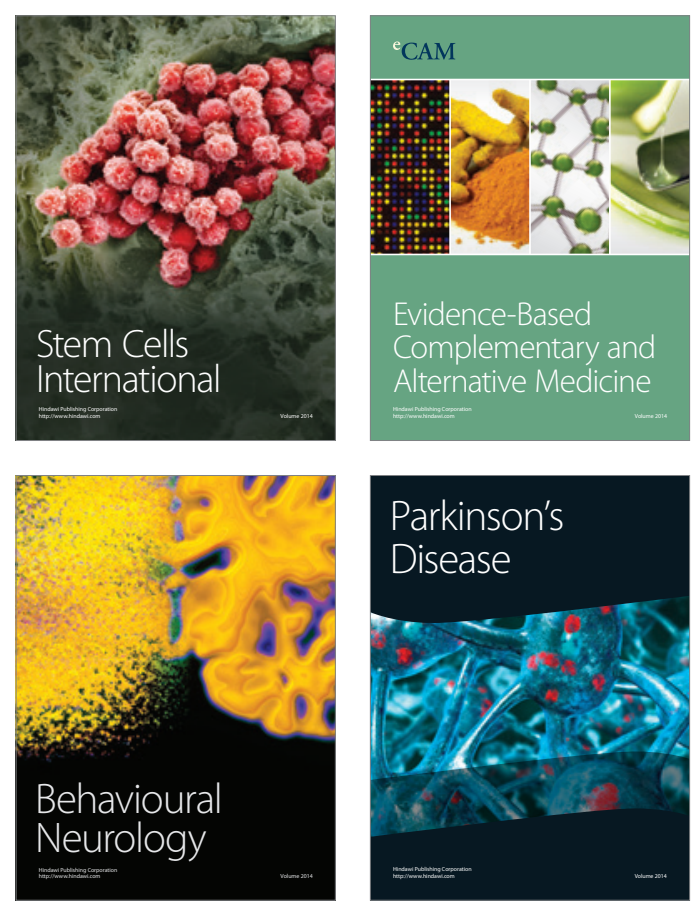

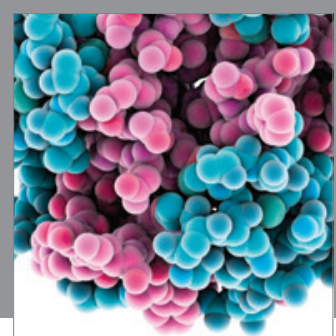

Journal of
Diabetes Research

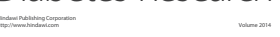

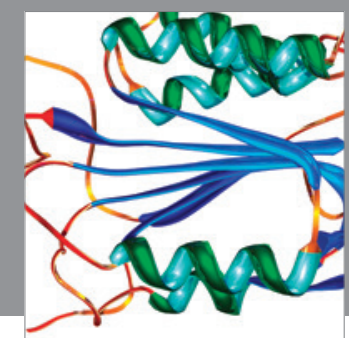

Disease Markers
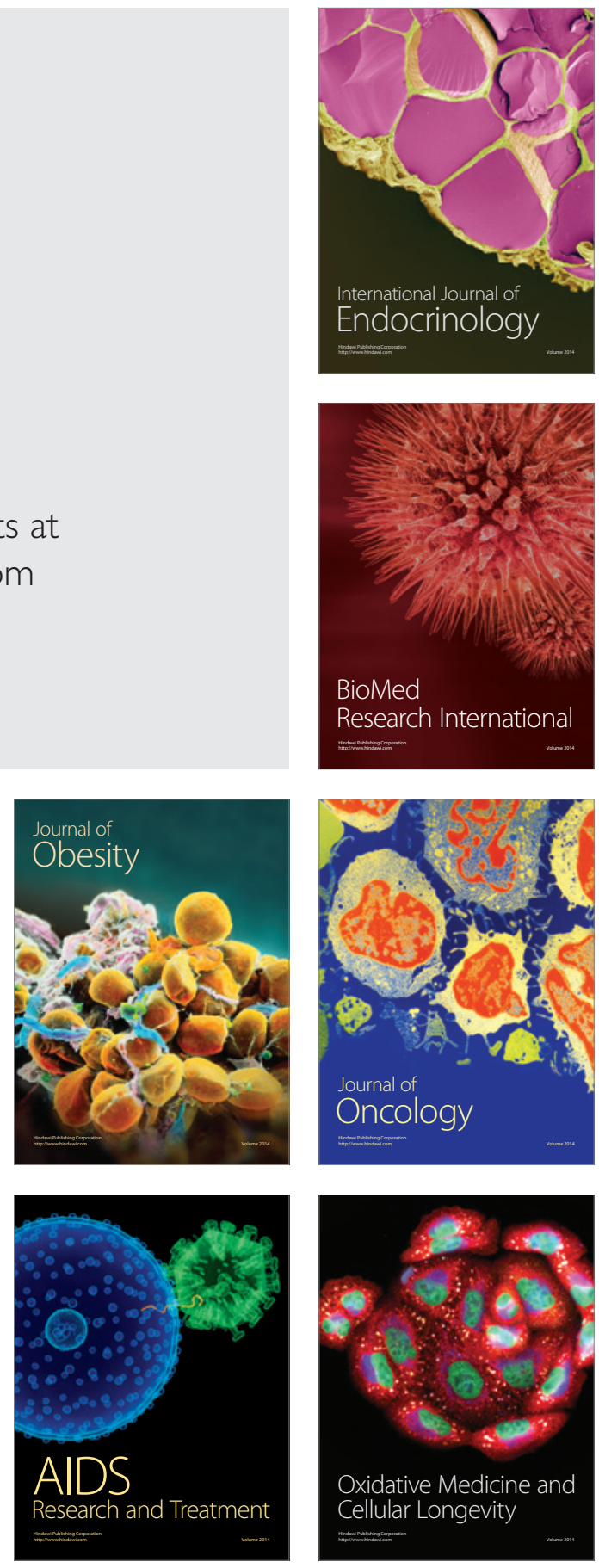